\title{
Efeitos adversos dos medicamentos antiparkisonianos e sua influência na qualidade de vida dos pacientes
}

\author{
Adverse effects of antiparkisonian medicines and their influence on the quality of life of \\ patients
}

Efectos adversos de los medicamentos antiparque y su influencia en la calidad de vida de los pacientes

lane Lopes da Rocha ${ }^{1 *}$, Emanuella de Castro Negreiros Nogueira ${ }^{1}$, Daniele Cavalcante Medeiros da Cunha ${ }^{1}$, Bruno Mendes Moura Dias Guerra ${ }^{1}$, Jackeline Dias Cunha Nogueira ${ }^{1}$, Andrey Sady de Sousa Almeida ${ }^{1}$, Kalliny Luiza Dias Arouca ${ }^{1}$, Thaiz Helena Lopes da Rocha ${ }^{1}$, Alyne Rodrigues de Araujo², Mário Abel Lima Barros ${ }^{1}$.

\section{RESUMO}

Objetivo: Avaliar aspectos relacionados à saúde mental e física do paciente com Parkinson e o impacto dos efeitos adversos do tratamento medicamentoso na qualidade vida. Métodos: Foi realizado um estudo transversal, não experimental e descritivo de abordagem quantitativa que utilizará como instrumento de pesquisa a escala unificada de avaliação da Doença de Parkinson (Unified Parkinsons' disease Rating Scale - UPDRS-ADAPTADA), com assinatura prévia dos pacientes e/ou responsável do Termo de Consentimento Livre e Esclarecido. Resultados: A amostra foi constituída por 20 pacientes acometidos pela doença de Parkinson e em uso de medicações antiparkinsonianas. Em geral, estes pacientes sentem-se tristes, mas não são diagnosticados com depressão e não possuem pensamentos suicidas. Em relação ao cotidiano, eles percebem uma lentidão, no entanto não se sentem incapazes e a maioria consegue realizar as tarefas diárias sem ajuda. Dor nas discinesias e distonias são os efeitos colaterais mais relatados pelos pacientes entrevistados. Conclusão: A partir desse estudo foi possível observar que o tratamento farmacológico pode contribuir para melhoria da qualidade de vida dos entrevistados e que os resultados encontrados corroboram com estudos já publicados sobre pacientes de outras regiões.

Palavras-chave: Qualidade de vida. Doença de Parkinson. Efeitos colaterais. Fármacos.

\begin{abstract}
Objective: To evaluate aspects related to the mental and physical health of parkinson's patients and the impact of adverse effects of drug treatment on quality of life. Methods: A cross-sectional, non-experimental and descriptive study of quantitative approach was carried out, using as a research instrument the unified parkinsons' disease rating scale (UPDRS-ADAPTED), with prior signature of patients and/or guardian of the Informed Consent Form. Results: The sample consisted of 20 patients affected by Parkinson's disease and using antiparkinsonian medications. In general, these patients feel sad, but are not diagnosed with depression and do not have suicidal thoughts. In relation to daily life, they perceive a slowness, however they do not feel incapable and most can perform daily tasks without help. Pain in dyskinesias and dystoniaare the side effects most reported by the interviewed patients. Conclusion: From this study it was possible to observe that pharmacological treatment can contribute to improve the quality of life of the interviewees and that the results found corroborate studies already published on patients from other regions.
\end{abstract}

Keywords: Quality of life, Parkinson's disease, Drug-related side effects, Drug.

\footnotetext{
${ }^{1}$ Instituição de Educação Superior do Vale do Parnaíba (IESVAP), Parnaíba - PI.

*E-mail: iane_Irocha@hotmail.com

2 Universidade Federal do Delta do Parnaíba (UFDPar), Parnaíba - PI.

3 Universidade Federal do Piauí. Teresina - PI.
} 


\section{RESUMEN}

Objetivo: Evaluar aspectos relacionados con la salud mental y física de los pacientes de parkinson y el impacto de los efectos adversos del tratamiento farmacológico en la calidad de vida. Métodos: Se llevó a cabo un estudio transversal, no experimental y descriptivo del enfoque cuantitativo, utilizando como instrumento de investigación la escala unificada de clasificación de la enfermedad de parkinson (UPDRSADAPTED), con la firma previa de los pacientes y/o tutor del Formulario de Consentimiento Informado. Resultados: La muestra consistió en 20 pacientes afectados por la enfermedad de Parkinson y el uso de medicamentos antiparkinsonianos. En general, estos pacientes se sienten tristes, pero no son diagnosticados con depresión y no tienen pensamientos suicidas. En relación con la vida diaria, perciben una lentitud, sin embargo no se sienten incapaces y la mayoría puede realizar tareas diarias sin ayuda. El dolor en las discinesias y distonías son los efectos secundarios más reportados por los pacientes entrevistados. Conclusión: A partir de este estudio fue posible observar que el tratamiento farmacológico puede contribuir a mejorar la calidad de vida de los entrevistados y que los resultados encontrados corroboran los estudios ya publicados en pacientes de otras regiones.

Palabras clave: Calidad de vida, Enfermedad de Parkinson, Efectos colaterales, Droga.

\section{INTRODUÇÃO}

A doença de Parkinson (DP) é caracterizada como um distúrbio neurodegenerativo na qual ocorre a morte gradual de neurônios localizados no núcleo do mesencéfalo denominado substância negra, resultando na diminuição da produção de dopamina (GARRET C e PEREIRA D, 2009; MAGALHÃES GHF, 2014). O sistema dopaminérgico e os neurônios de melanina sofrem despigmentação. Assim, quanto mais clara for à substância negra, maior será a perda de dopamina. (MENOTTI LD e ZANUSSO JUNIOR G, 2015).

Essa redução causa uma degradação da via nigroestriatal, causando uma oscilação de neurotransmissores excitatórios e inibitórios no corpo estriado. Com isso, há um comprometimento extrapiramidal quanto ao domínio dos movimentos corporais complexos, desenvolvendo tremores, rigidez, bradicinesia e instabilidade postural (ANDERSON C e BRAUN C, 2009).

A substância negra localiza-se dorsalmente ao pedúnculo e ventralmente ao tegumento, se estendendo longitudinalmente ao mesencéfalo. É caracterizada por sua pigmentação escura devido a uma grande quantidade de melanina. Divide-se em duas partes: a compacta e a reticular (MENESES M, 2006).

A parte compacta é formada por células volumosas e pigmentadas. Seus neurônios são ricos em dopamina, o que os caracterizam como a principal fonte de dopamina estriatal (núcleo caudado e putâmen) (MENESES M, 2006).

As fibras aferentes nigrais saem do segmento lateral do globo pálido, do núcleo subtalâmico, do núcleo dorsal da rafe, do núcleo pedúnculo-pontino e principalmente do núcleo caudado e putâmen (neoestriado), sendo, estas últimas, conhecidas como fibras estriatonigrais (MENESES M, 2006). As fibras eferentes nigrais surgem das duas partes da substância negra, tendo neurotransmissores e projeções diferentes: fibras nigroestriatais (neurônios dopaminérgicos), fibras nigrotalâmicas e nigrotegmentares (neurônios GABAérgicos) (MENESES M, 2006).

Funcionalmente, as principais fibras aferentes e eferentes são as que se conectam com o corpo estriado (fibras nigroestriatais e estriatonigrais). Essa destruição dos neurônios dopaminérgicos da substância negra causa uma depleção de dopamina no corpo estriado, e com isso, graves alterações motoras são provocadas, o que caracteriza a Síndrome de Parkinson (MENESES M, 2006).

A sintomatologia da patologia é caracterizada por distúrbios motores, cognitivos e emocionais que se intensificam com a progressão da doença. Entretanto, os sinais e sintomas motores característicos da doença só passam a ser visíveis clinicamente quando já houve a degeneração de mais de $60 \%$ dos neurônios dopaminérgicos da via nigro-estriatal (SAKHARKAR MK, et al., 2019). 
A princípio, as alterações motoras apresentam-se com predomínio unilateral, evoluem para manifestações bilaterais e em estágios mais avançados acarretam perda da autonomia funcional (MANCOPES R, et al., 2013).

Dentre os sintomas motores, o mais característico é o tremor, que resulta da contração alternada de músculos agonistas e antagonistas cuja característica pode ser descrita como um tremor lento e ocorre de forma mais nítida quando os membros estão em repouso, desaparecendo durante o sono. A rigidez na DP diverge da rigidez causada por lesões dos neurônios motores superiores no fato de que está presente na mesma extensão em grupos de músculos opostos. Na bradicinesia, observa-se tanto a dificuldade em iniciar movimentos quanto de executar movimentos novos. Já em relação à postura, o paciente permanece em pé numa posição encurvada, com os braços fletidos. (SNELL RS, et al., 2019).

Esse conjunto de sinais e sintomas é essencial para o diagnóstico da doença, visto que não há testes laboratoriais e marcadores biológicos que a confirmem. Portanto, utiliza-se a tétrade: acinesia ou bradicinesia, rigidez muscular, tremor de repouso e instabilidade postural, como critérios fundamentais para classificação da DP. Diante disso, a presença de dois ou quatro desses sinais sugere o diagnóstico clínico da doença (SAITO T, 2011).

É importante destacar as manifestações não motoras que também estão presentes como: a depressão, demência e a ansiedade. Essas alterações estão diretamente relacionadas com a evolução dos sintomas físicos e cognitivos, que incapacitam o autocuidado. Por consequência há uma piora na qualidade de vida do paciente. Na contemporaneidade a DP é considerada a segunda maior e mais prevalente doença neurológica senil. Estima-se uma prevalência de 100 a 200 casos por 100 mil habitantes (MENDES F, 2017).

Dados da Organização Mundial de Saúde (OMS) revelam que 1\% da população acima dos 65 anos sofre com o Mal de Parkinson. A DP não apresenta predisposição por classes sociais, raça, cor e sexo. Segundo a Portaria № 228 do Ministério da Saúde, a Doença de Parkinson é uma doença de distribuição universal e atinge todos os grupos étnicos e classes socioeconômicas. Ainda não foi descoberta a definitiva etiologia da DP, no entanto muitas hipóteses são elencadas. A mais notável, é a causa multifatorial, ou seja, a combinação da tendência genética, fatores ambientais, distúrbios metabólicos, anormalidades celulares ou ainda, modificações do envelhecimento (SOUZA CP, et al., 2016; BEKRIS LM, et al.,2010).

O tratamento do Parkinson não visa à cura do paciente, pois ainda não se conhece medicamento que faça recuperar ou regredir a degeneração neuronal. A maior importância durante o período da enfermidade é estender ao máximo que o paciente tenha autonomia de suas funções (MARCHI KC, et al., 2013).

Os medicamentos, em geral, aliviam os sintomas causados pela doença, mas é de bastante relevância alertar sobre as consequências que o uso crônico dessas drogas causa, avaliando e, se precisar, reajustar o esquema posológico do paciente na tentativa de um equilíbrio entre o alívio dos sintomas e a prevenção de reações adversas indesejáveis. As principais drogas utilizadas são: levodopa, selegilina, bromocriptina, pramipexol, tolcapone e amantadina (LOPES MA, et al., 2012). A levodopa é um antiparkinsoniano precursor imediato de dopamina. Ela ultrapassa a barreira hematoencefálica e é quebrada pela dopa descarboxilase em dopamina nos neurônios nigroestriatais. Assim, a quantidade do neurotransmissor deficiente é restaurada (SILVA P, 2010).

Apesar de reduzir os sintomas da doença, a levodopa causa efeitos colaterais em alguns sistemas do corpo do paciente. No trato gastrointestinal causa, em sua grande maioria, anorexia, náuseas, vômitos e desconforto epigástrico na fase inicial, devendo, por causa disso, ser administrada juntamente com inibidores periféricos de descarboxilase (SILVA P, 2010). Apesar de não ser muito frequente, é necessário estar atento aos pacientes com problemas cardíacos, pois arritmias cardíacas já foram descritas devido ao uso da levodopa. Além disso, os pacientes podem apresentar hipotensão ortostática no início do tratamento, o que melhora com a continuidade do tratamento (SILVA P, 2010).

Alterações mentais aparecem em uma quantidade pequena de pacientes, e isso inclui agitação, confusão mental, alucinações, ilusões, ideias paranoides, sonhos vívidos, hipomania, depressão, conduta hipersexual e psicoses (SILVA P, 2010). 
Os distúrbios neurológicos são muitos, as discinesias, por exemplo, podem aparecer logo depois dos primeiros meses de tratamento com a levodopa. Esses movimentos involuntários são dependentes da dose e reduzem com a diminuição da dosagem do medicamento (SILVA P, 2010).

Com o tratamento continuado principalmente por mais de seis anos, ainda pode acontecer o que se chama de fenômeno on-off (liga-desliga). O paciente fica em um estado de acinesia, pausa, não consegue se movimentar seguido por um momento de retorno dos movimentos (SILVA P, 2010). Grande parte dos pacientes que utilizam a levodopa têm os efeitos colaterais referentes ao tempo e à dose do tratamento. $A$ eficácia desse medicamento tem seu declínio depois de 3 a 5 anos (SILVA P, 2010). A selegilina é outro tipo de medicamento, ele é um inibidor seletivo da monoamina oxidase tipo $B$, enzima que quebra a dopamina no cérebro, portanto é responsável pela inibição da degradação intraneural de dopamina no sistema nigroestriatal e bloqueador da recaptação dessa substância (SILVA P, 2010).

Quando utilizada junto com a levodopa o efeito dopaminérgico aumenta, e dessa forma pode causar boca seca, mal-estar intestinal, vertigem, confusão mental, alucinações, câimbras, insônia, hipotensão ortostática e arritmias cardíacas (SILVA P, 2010). A bromocriptina tem como mecanismo de ação estimular os receptores dopaminérgicos do SNC, do sistema cardiovascular, eixo hipotálamo-hipofisário e trato gastrointestinal (SILVA P, 2010). Seus efeitos colaterais são náuseas, tonturas, vômitos, fadiga, hipotensão ortostática, constipação, sonolência e boca seca. Porém, tem uma vantagem em relação à levodopa, pois tem menos predisposição a apresentar discinesias e o fenômeno on-off (SILVA P, 2010).

O pramipexol é um agonista de dopamina de grande seletividade e afinidade pelo receptor D2 de dopamina. Os pacientes que fazem uso desses medicamentos geralmente reclamam de náuseas, constipação, tontura, sonolência, insônia, astenia, alucinações, hipotensão postural, cefaleia, confusão e discinesias (SILVA P, 2010).

O tolcapone é um inibidor reversível e seletivo da catecol-O-metil-transferase (COMT), enzima muito importante no catabolismo da levodopa, central e periférico, o que eleva a levodopa periférica, aumentando sua biodisponibilidade (SILVA P, 2010). Os efeitos colaterais que mais aparecem são anorexia, náuseas, diarreia, dispepsia, hipotensão postural, tontura, cefaleia, sonolência leve, transtorno do sono, discinesia e a disfunção hepática (SILVA P, 2010).

O tratamento para o Mal de Parkinson objetiva a manutenção da qualidade de vida do paciente, com o alcance almejado da autoestima e a sua reinserção social, assegurando-Ihe o direito de cidadania (MANDIRA $A, 2012)$. O conceito de qualidade de vida indica as condições de vida de um ser humano, que envolve várias áreas, como o bem físico, mental, psicológico e emocional, relacionamentos sociais, como família e amigos e também saúde, educação e outros parâmetros que afetam a vida humana (ALMEIDA MAB, et al., 2012).

Estudos comprovam que as quatro principais características que atingem indivíduos com DP são o tremor de repouso, a rigidez, a bradicinesia (lentidão nos movimentos) e a instabilidade postural. Outra característica clínica clássica da DP é a hipocinesia (diminuição dos movimentos). Além disso, são comumente acompanhadas por fadiga, congelamento da marcha, depressão, sintomas autonômicos, dor e, muitas vezes, demência. Esses fatores juntos, ou mesmo sozinhos, podem elevar o risco de quedas, restringindo o desenvolvimento de atividades e diminuindo a participação social desses indivíduos (SILVA D, 2015).

Neste contexto, o vigente estudo tem por objetivo analisar a eficácia do tratamento farmacológico e os efeitos adversos na melhoria da qualidade de vida dos pacientes com Parkinson.

\section{MÉTODOS}

Trata de um estudo transversal, não experimental e descritivo de abordagem quantitativa, que foi realizada no período de fevereiro de 2018 a março de 2019. A pesquisa foi conduzida por buscas através dos termos livres "Bem-estar" (Wellness), "Doença de Parkinson" (Parkinson Disease), "Efeitos colaterais" (Side effects) e Medicação (Medication), nas seguintes bases de dados eletrônicas, Literatura Latino-americana e do Caribe em Ciências da Saúde (Lilacs), Scientific Electronic Library Online (Scielo), Medical Literature Analysis and Retrieval System Online (Medline/Pubmed), no período de 2006 a 2017. 
Um total de 75 artigos que preenchiam os requisitos propostos pela metodologia da pesquisa foram analisados para complementação dos dados. A amostra conta com 45 pacientes em cadastro ativo na farmácia de componente especializado que abrange as cidades da planície litorânea do Piauí. O projeto foi aprovado pelo comitê de ética em pesquisa do grupo ITPAC.

Os critérios de inclusão dos pacientes foram: diagnóstico confirmado pelo neurologista de DP, aprovação do paciente ou responsável por meio do Termo de Consentimento Livre e Esclarecido, estar cadastrado e ativo na Farmácia Componente Especializado da Planície Litorânea.

Os dados foram colhidos no período de fevereiro a março de 2019, por três pesquisadores durante entrevista individual. Foi utilizado um questionário (a escala unificada de avaliação da Doença de Parkinson (Unified Parkinsons' disease Rating Scale - UPDRS- ADAPTADA), a fim de analisar a que ponto os efeitos adversos das medicações interferem na qualidade de vida dos pacientes. As variáveis foram resumidas em tabelas e gráficos mediante frequência absoluta e relativa (porcentagem). Os gráficos foram construídos com auxílio dos programas Exce ${ }^{\circledR}$ e GraphPad Prism 6.0.

\section{RESULTADOS}

A amostra foi constituída por 20 pacientes que são acometidos pela doença de Parkinson e em uso de medicações antiparkinsonianas, usuários da rede de saúde da Planície Litorânea - PI. Em relação ao estado mental, a maioria dos pacientes (70\%) afirmou apresentar períodos de tristeza ou culpa acima do normal, entretanto esses períodos não são duradouros. Nenhum paciente relatou a existência de pensamentos suicidas (Tabela 1).

Tabela 1 - Distribuição de pacientes quanto ao estado mental, comportamento ou estado emocional do paciente em relação à depressão.

\begin{tabular}{lcc}
\hline Depressão & $\begin{array}{c}\text { Frequência } \\
\text { absoluta (fi) }\end{array}$ & Frequência relativa (fri \%) \\
\hline Ausente & 5 & $25 \%$ \\
\hline $\begin{array}{l}\text { Períodos de tristeza ou culpa acima do normal. Nunca } \\
\text { permanece por dias ou semanas. }\end{array}$ & 14 & $70 \%$ \\
\hline $\begin{array}{l}\text { Depressão permanente com sintomas vegetativos } \\
\text { (insônia, anorexia, perda de peso, desinteresse). }\end{array}$ & 1 & $5 \%$ \\
\hline $\begin{array}{l}\text { Depressão permanente com sintomas vegetativos. } \\
\text { Pensamento ou tentativa de suicídio. }\end{array}$ & 0 & $0 \%$ \\
\hline Total & 20 & $100 \%$ \\
\hline
\end{tabular}

Fonte: Rocha IL, et al., 2020.

Quanto à motivação dos pacientes usuários de medicamentos antiparkinsonianos, 70\% apresentam-se normais, enquanto $15 \%$ sentem-se mais passivos, ou seja, menos interessados que o habitual, ou com desinteresse por atividades diferentes daquelas rotineiras (Tabela 2).

Tabela 2 - Distribuição de pacientes quanto ao estado mental, comportamento ou estado emocional do paciente em relação à motivação/iniciativa.

\begin{tabular}{lcc}
\hline Motivação/iniciativa & $\begin{array}{c}\text { Frequência } \\
\text { absoluta (fi) }\end{array}$ & Frequência relativa (fri \%) \\
\hline Normal & 14 & $70 \%$ \\
\hline Mais passivo, menos interessado que o habitual. & 3 & $15 \%$ \\
\hline $\begin{array}{l}\text { Perda da iniciativa ou desinteresse por atividades } \\
\text { fora do dia-a-dia. }\end{array}$ & 3 & $15 \%$ \\
\hline Total & 20 & $100 \%$ \\
\hline
\end{tabular}

Fonte: Rocha IL, et al., 2020. 
Uma parcela significativa dos pacientes entrevistados relata um comprometimento superficial na fala (45\%), pouca lentidão na escrita (50\%), na manipulação dos alimentos $(45 \%)$, no ato de se vestir (55\%) e no momento de realizar a higiene pessoal (70\%), quando afirmam não precisar de ajuda. Além de conseguirem realizar as atividades diárias, ainda que com um pouco de lentidão, muitos pacientes (55\%) afirmam não sofrerem quedas devido ao "freezing" característico da doença de Parkinson, que aparece raramente no início da marcha de $45 \%$ dos entrevistados.

Em relação à marcha, metade dos pacientes apresenta uma pequena dificuldade, podendo não balançar os braços ou tendendo a arrastar as pernas, enquanto $25 \%$ deles conseguem caminhar normalmente. Da mesma forma, metade dos pacientes apresentam tremores, mas sem muita frequência. No entanto, $30 \%$ dos pacientes $(n=6)$ apresentam tremores moderados que incomodam (Figura 1). Apenas um paciente (5\%) relata ausência dos tremores com o uso da medicação.

Figura 1 - Distribuição de pacientes quanto ao tremor.

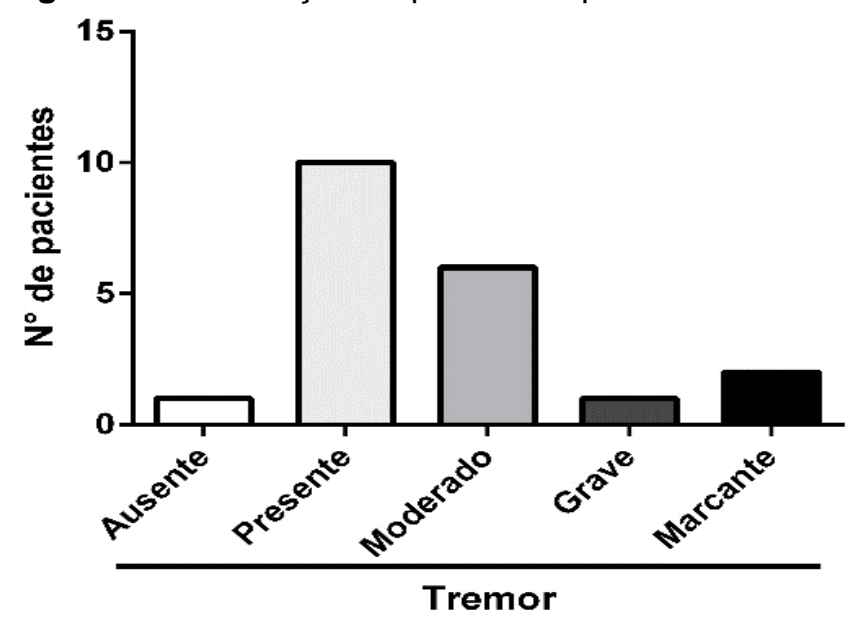

Fonte: Rocha IL, et al., 2020.

A discinesia, como complicação da terapia, foi reportada por $75 \%$ dos pacientes, destes, a maioria sente por um período de 6 horas por dia ou 25\% do dia (Figura 2).

Figura 2 - Percentual de pacientes que reportaram discinesia como complicação da terapia para tratamento do Parkinson.

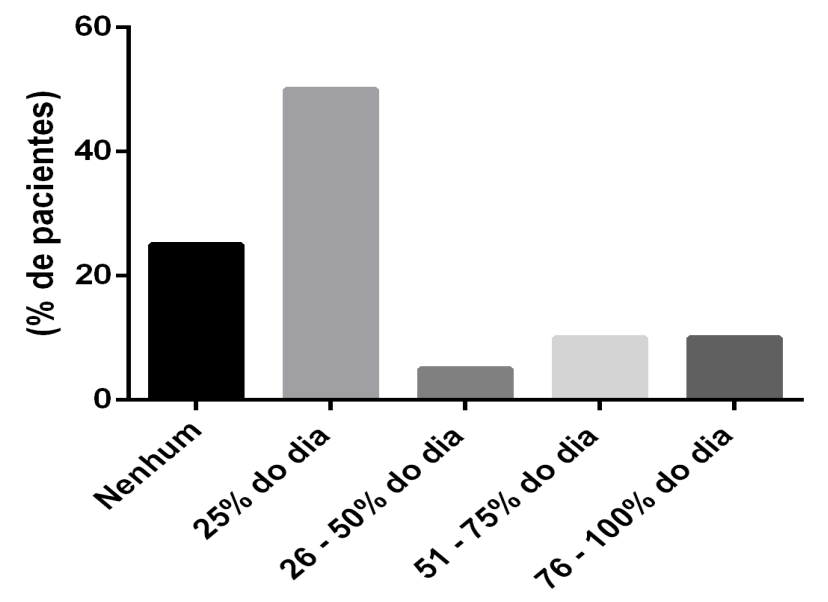

Fonte: Rocha IL, et al., 2020. 
A incapacidade também foi abordada pelos entrevistados como complicação da terapia. Em relação a este parâmetro, $65 \%$ dos pacientes não demonstraram incapacidade e $35 \%$ apresentaram incapacidade de leve a grave (Tabela 3).

Tabela 3 - Distribuição de pacientes quanto à incapacidade como complicação da terapia.

\begin{tabular}{lcc}
\hline Incapacidade & $\begin{array}{c}\text { Frequência } \\
\text { absoluta (fi) }\end{array}$ & $\begin{array}{c}\text { Frequência } \\
\text { relativa (fri \%) }\end{array}$ \\
\hline Não incapacitante & 13 & $65 \%$ \\
Incapacidade leve & 3 & $15 \%$ \\
Incapacidade moderada & 2 & $10 \%$ \\
Incapacidade grave & 2 & $10 \%$ \\
Completamente incapaz & 0 & $0 \%$ \\
\hline Total & 20 & $100 \%$ \\
\hline
\end{tabular}

Fonte: Rocha IL, et al., 2020.

As outras complicações da terapia, como anorexia, náusea ou vômitos, distúrbio do sono, hipotensão ortostática e nictúria foram relatadas pela maioria dos pacientes, enquanto complicações tais como constipação, urgência miccional, perda de olfato ou paladar e sudorese excessiva, são reportadas pela minoria dos pacientes (Figura 3 e Figura 4). Assim como 60\% dos pacientes que afirmaram não apresentar distonias.

Figura 3 - Complicações neurológicas, gastrointestinais e pressóricas.

\section{ANOREXIA, NÁUSEA OU VÔMITOS DISTÚRBIO DO SONO}

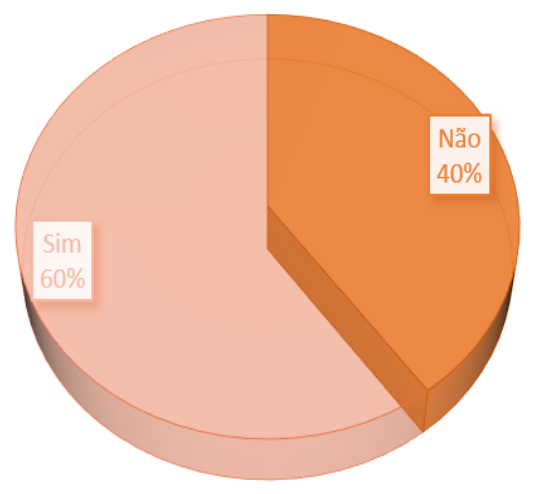

HIPOTENSÃO ORTOSTÁTICA

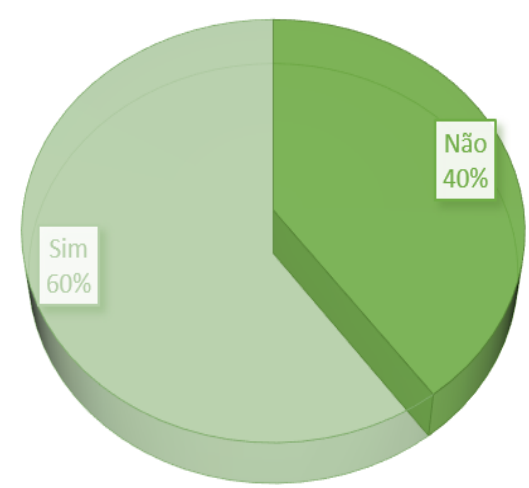

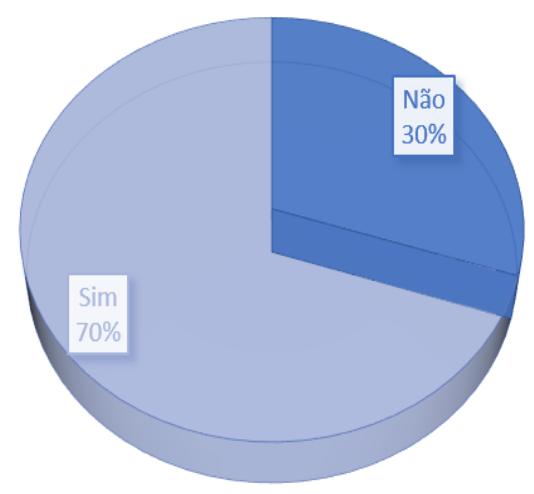

CONSTIPAÇÃO

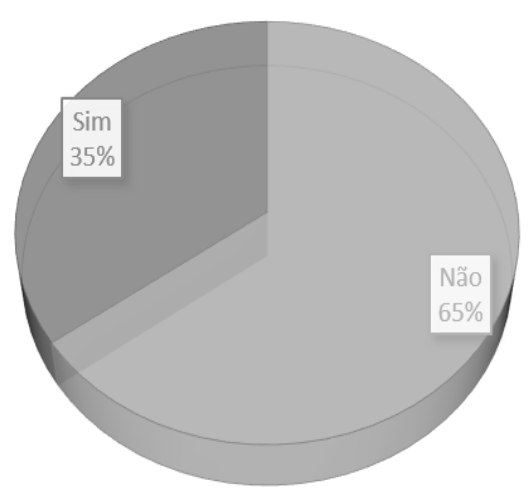

Fonte: Rocha IL, et al., 2020. 
Figura 4 - Complicações urinárias, anormalidade sensoriais e perdas insensíveis.

\section{URGÊNCIA MICCIONAL}

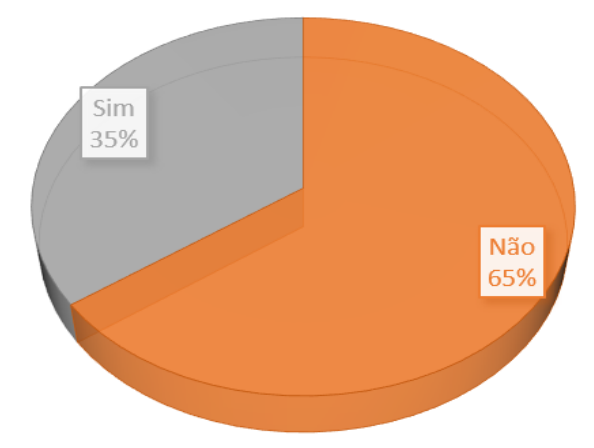

PERDEU OLFATO OU PALADAR

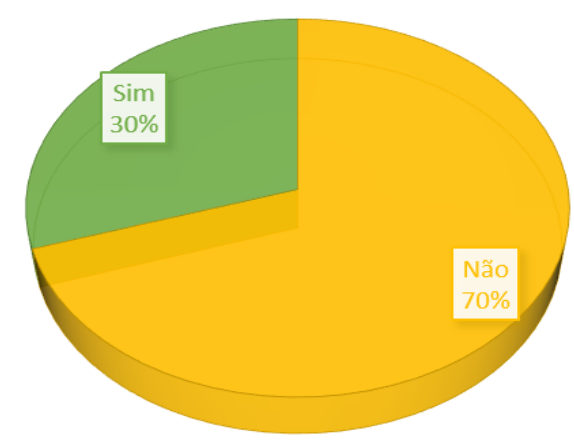

Fonte: Rocha IL, et al., 2020.

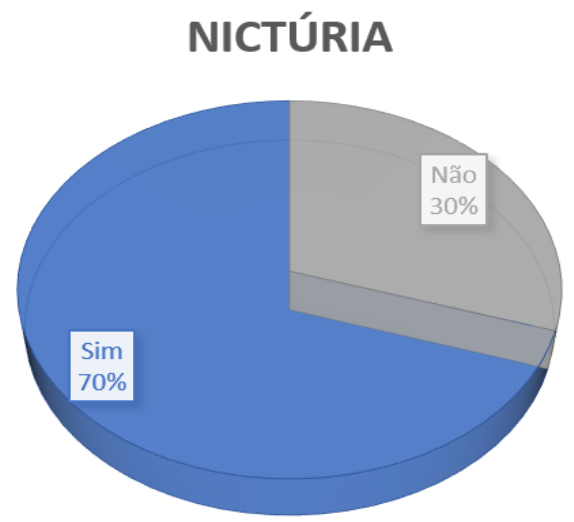

SUDORESE EXCESSIVA

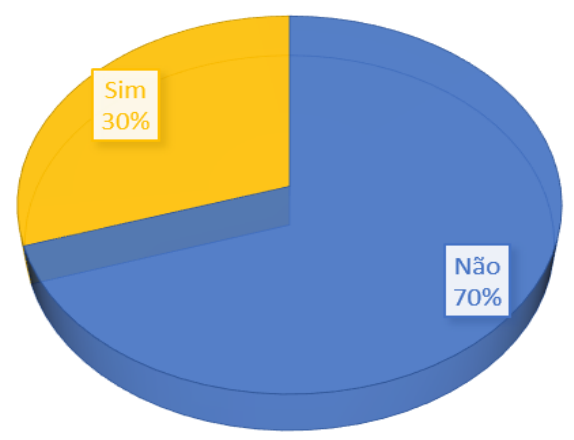

\section{DISCUSSÃO}

Os resultados, obtidos por meio dos questionários aplicados aos pacientes usuários de medicamentos para tratamento da doença de Parkinson, permitiram avaliar aspectos relacionados ao estado mental e ao cotidiano do paciente, bem como os sintomas característicos da doença e aqueles que aparecem em decorrência do uso contínuo das drogas antiparkinsonianas. A doença de Parkinson é uma doença neurodegenerativa multissistêmica, comum no Brasil. Os transtornos mentais e alterações relacionadas ao comportamento são frequentes em portadores desta doença, tais transtornos prejudicam a qualidade de vida dos pacientes que, muitas vezes, apresentam ansiedade, depressão, psicose, dentre outros (KUMMER A e TEIXEIRA AL, 2009).

Pesquisas que avaliam a cognição em transtorno bipolar e unipolar do humor (depressão maior) constatam que há disfunção executiva e de memória de trabalho, além de deficiência de memória, tanto na fase clínica como na assintomática (SILBERMAN C, et al., 2004).

O tratamento prévio e eficaz na depressão tem resultado positivo sobre o desenvolvimento cognitivo dos parkinsonianos, o que torna de extrema importância à necessidade eventual de reconhecimento da sintomatologia depressiva para a melhoria desses pacientes. Além disso, o diagnóstico precoce de depressão pode dirimir dúvidas sobre a ocorrência de demência na evolução da doença de Parkinson, uma vez que esse diagnóstico diferencial nem sempre é simples (SILBERMAN C, et al., 2004).

A maioria dos pacientes desse estudo sente-se triste, no entanto os pacientes relatam que essa tristeza não é duradoura e que não ocorrem pensamentos suicidas, enquanto apenas $5 \%$ da população entrevistada relatou depressão. Esse percentual ficou abaixo dos 14\% relatado por (ALMEIDA L, et al. 2016) para a população da Flórida (EUA). Além de distúrbios neurológicos, os pacientes podem apresentar também alterações relacionadas à comunicação, principalmente, decorrentes da limitação dos movimentos dos 
músculos associados aos mecanismos da fala. Sabe-se que a medicação utilizada para o tratamento do Parkinson pode produzir efeito variável sobre as alterações que a doença ocasiona, assim, este estudo demonstra que a maioria dos pacientes relata alteração na fala de superficial a moderada (BARROS AL, et al., 2004; MURDOCH B, et al., 2011).

A cronicidade da doença de Parkinson proporciona mudanças no cotidiano dos pacientes, no que diz respeito à sua individualidade e capacidade de realização das tarefas diárias, em sua fase mais avançada, tende a paralisar os músculos, acarretando, com isso, maior lentidão para desenvolver atividades do dia a dia. Nesse sentido, a medicação representa algo positivo quando há percepção de suas vantagens na redução dos sinais e sintomas decorrentes do quadro clínico do paciente (VALCARENGHI RV, et al., 2018). Esse aspecto pode ser observado nos resultados encontrados nesse estudo, que demonstram, no geral, uma lentidão em atividades da vida diária. Entretanto, vale ressaltar, que poucos pacientes necessitam de ajuda para realizar suas atividades diárias.

Quanto ao tratamento medicamentoso, ainda não existem fármacos capazes de interromper o curso da doença nem de preveni-las; os existentes têm como objetivo o controle dos sintomas no intuito de manter o portador com autonomia, independência funcional e equilíbrio psicológico (GONÇALVES LHT, et al., 2007). A literatura reporta que o tratamento medicamentoso da doença de Parkinson com levodopa, pramipexol, metilfenidato e inibidores da monoaminoxidase podem ajudar a diminuir o freezing da marcha. Esse dado corrobora com os achados nesse estudo, uma vez que muitos pacientes afirmam apresentar o freezing raramente e nenhum paciente relatou ter caído em decorrência do freezing (ZHANG LL, et al., 2016).

Em relação aos sintomas motores do Parkinson, os tremores e a discinesia podem ser considerados os sintomas mais importantes e diminuem a qualidade de vida dos pacientes. Estudos apontam para uma tendência em aumentar esses sintomas mediante o tratamento prolongado com levodopa, que é o padrão ouro para o tratamento de desordens motoras em associação com outros fármacos. Metade dos pacientes desse estudo afirmaram apresentar discinesia em $25 \%$ do dia $(6 \mathrm{~h})$ e tremores (ainda que não sejam frequentes), dessa forma, acredita-se que tratamentos alternativos aliados ao tratamento medicamentoso podem reduzir a incidência desses sintomas motores (DANEAULT JF, et al., 2013).

Os efeitos colaterais mais prevalentes dentre os entrevistados foram dor nas discinesias, presença de distonias, anorexia, náuseas, vômitos, distúrbios do sono, hipotensão ortostática e nictúria. A maioria desses efeitos colaterais já são relatados na literatura como mais prevalentes, reforçando os resultados desse estudo (VALCARENGHI RV, et al., 2018). Mesmo sabendo que ainda não há cura para a doença de Parkinson, é importante ressaltar que as manipulações farmacológicas são eficazes no alívio sintomático da doença.

\section{CONCLUSÃO}

A partir dos dados compilados nesse estudo foi possível observar que o tratamento farmacológico realizado pelos pacientes com Parkinson pode contribuir para melhoria da qualidade de vida dos pacientes entrevistados, que apresentaram poucos distúrbios neurológicos. A análise dos dados também permitiu concluir que os pacientes da Planície Litorânea - PI apresentam alguns sintomas predominantes, provavelmente decorrente de efeitos colaterais, tais como distonias e dor nas discinesias, corroborando com estudos já publicados sobre pacientes de outras regiões.

\section{REFERÊNCIAS}

1. ALMEIDA L, et al. Depressive Symptoms are Frequent in Atypical Parkinsonian Disorders. Mov Disord Clin Pract, 2016; 4(2): 191-197.

2. ALMEIDA MAB, et al. Qualidade de Vida: definição, conceitos e interfaces com outras áreas de pesquisa. Escola de Artes, Ciências e Humanidades - EACH/USP. São Paulo, 2012.

3. ANDERSON C, BRAUN C. Fisiopatologia: Alterações funcionais na saúde humana. 1.ed. Porto Alegre: Artmed. 2009.

4. BARROS AL, et al. Uma análise do comprometimento da fala em portadores de doença de Parkinson. Revista Neurociências, 2004; 12(3): 123-129.

5. BEKRIS LM, et al. The genetics of Parkinson disease. J Geriatr Psychiatry Neurol, 2010; 23(4): $228-242$. 
6. DANEAULT JF, et al. Drug-induced dyskinesia in Parkinson's disease. Should success in clinical management be a function of improvement of motor repertoire rather than amplitude of dyskinesia? BMC Medicine, 2013; 11: 76-93.

7. GARRETT C, PEREIRA D. Factores de risco da doença de Parkinson um estudo epidemiológico. Acta Méd Port, 2010; 23: 15-24.

8. GONÇALVES LHT, et al. Pacientes portadores da doença de Parkinson: significado de suas vivências. Acta paul. enferm., 2007; 20(1): 62-68.

9. KUMMER A, TEIXEIRA AL. Neuropsychiatry of Parkinson's Disease. Arq Neuropsiquiatr, 2009; 67(3-B): $930-939$.

10. LOPES MA, et al. Reações adversas causadas por antiparkinsonianos. Interfaces Científicas - saúde e ambiente, 2012; 1(1).

11. MAGALHÃES GHF. Tecnologias Cognitivas: Uma Abordagem Geral. IV Seminário Interno de Cognição Artificial SICA - FEEC - UNICAMP, 2014.

12. MANCOPES R, et al. Influência da levodopa sobre a fase oral da deglutição em pacientes com doença de Parkinson. Rev CEFAG, 2013; 15(3): 707-712.

13. MANDIRA A. Estudo da influência positiva na qualidade de vida de portadores da Doença de Parkinson participantes do Grupo Lótus. Rev Kairós, 2012; 15(8): 185-199.

14. $\mathrm{MARCHI} \mathrm{KC}$, et al. Adesão à medicação em pacientes com doença de Parkinson atendidos em ambulatório especializado. Cien Saúde Colet, 2013; 18(3): 855-862.

15. MENDES F. Evolução da doença de Parkinson - operacionalização da decisão cirúrgica. 116f. Dissertação de candidatura ao grau de Doutor em Ciências Médicas. Portugal, Universidade do Porto, 2017.

16. MENESES M. Neuroanatomia aplicada. 2.ed. Rio de Janeiro: Guanabara Koogan, 2006.

17. MENOTTI L D, ZANUSSO JUNIOR G. Doença de Parkinson: uma revisão. Brazilian Journal of Surgery and Clinical Research - BJSCR. Paraná, 2015; 13(1): 70-77.

18. MURDOCH B, et al. Communication Impairments in Parkinson's Disease. Parkinson's Disease. 2011: 1-2.

19. SAITO T. A Doença de Parkinson e Seus Tratamentos: uma revisão bibliográfica. $35 f$. Tese do curso de Especialização em Saúde Coletiva e Saúde. Londrina, Centro Universitário Filadélfia, 2011.

20. SAKHARKAR MK, et al. A systems biology approach towards the identification of candidate therapeutic genes and potential biomarkers for Parkinson's disease. PLoS One. 2019.

21. SILBERMAN CD et al. Uma revisão sobre depressão como fator de risco na Doença de Parkinson e seu impacto na cognição. Rev. psiquiatr. Rio Gd. Sul, Porto Alegre, 2004; 26(1): 52-60.

22. SILVA D. Perfil dos indivíduos com doença de Parkinson atendidos no setor de fisioterapia de um hospital universitário no Rio de Janeiro. Braz J Psychiatry, 2015; 51(4): 100-105.

23. SILVA P. Farmacologia. 8.ed. Rio de Janeiro; Guanabara Koogan, 2010.

24. SNELL RS. Neuroanatomia clínica. Rio de Janeiro: Guanabara Koogan, 2019.

25. SOUZA CP, et al. The interlocking finger test in patients with Parkinson's disease and healthy subjects. $J$ Clin Neurosci, 2016; 29: 145-148.

26. VALCARENGHI RV, et al. O cotidiano das pessoas com a doença de Parkinson. The daily lives of people with Parkinson's disease. Rev Bras Enferm, 2018; 71(2): 272-279.

27. ZHANG LL, et al. Freezing of Gait in Parkinsonism and its Potential Drug Treatment. Curr Neuropharmacol, 2016; 14: 302-306. 